\title{
PEG-conjugated TLR7/8 Agonist NKTR-262
}

National Cancer Institute

\section{Source}

National Cancer Institute. PEG-conjugated TLR7/8 Agonist NKTR-262. NCI Thesaurus. Code C148289.

A formulation composed of an agonist of toll-like receptor (TLR) type 7 and 8 (TLR7/8) that is attached to polyethylene glycol (PEG) via a hydrolyzable glycine linker, with potential immunostimulating and antitumor activities. Upon intratumoral (IT) administration, TLR7/8 agonist NKTR-262 binds to and activates TLR7 and 8, thereby activating TLR7/8-mediated pathways. This stimulates the maturation and activation of antigen-presenting cells (APCs), including dendritic cells (DCs). Activation of DCs results in the production of pro-inflammatory cytokines, and the activation of cytotoxic Tlymphocyte (CTL)- and B-lymphocyte-mediated immune responses against tumorassociated antigens (TAAs), which lead to tumor cell lysis. TLR7 and 8, members of the TLR family, play fundamental roles in the activation of the innate immune system, myeloid cell responses and tumor antigen presentation. Pegylation of the TLR7/8 agonist enhances retention of this agent in the tumor microenvironment (TME), provides local sustained release of the TLR7/8 agonist, increases efficacy and reduces systemic exposure compared to the administration of the naked TLR7/8 agonist alone. 